\title{
Comparison between Splenic and Lymph Node Aspirations as Sampling Methods for the Parasitological Detection of Leishmania chagasi Infection in Dogs
}

\author{
Stella Maria Barrouin-Melo/*/+, Daniela Farias Larangeira, Joelma Trigo*, \\ Paulo Henrique Palis Aguiar/*, Washington Luís Conrado dos-Santos/**, \\ Lain Pontes-de-Carvalho /**
}

\begin{abstract}
Centro de Pesquisas Gonçalo Moniz-Fiocruz, Rua Valdemar Falcão 121, 40295-001 Salvador, BA, Brasil *Escola de Medicina Veterinária, Universidade Federal da Bahia, Salvador, BA, Brasil **Escola Bahiana de Medicina, Fundação para o Desenvolvimento das Ciências, Salvador, BA, Brasil
\end{abstract}

\begin{abstract}
The sensitivities of spleen and lymph node cultures for the diagnosis of canine visceral leishmaniasis were compared in 64 anti-Leishmania antibody positive dogs from an endemic area in Brazil. The sensitivity of spleen cultures for Leishmania detection was $97.9 \%$; in lymph node cultures it was $25 \%$. Positive spleen culture was more frequent ( $p=0.048$, Fisher's exact probability test) in symptomatic (28 out of 33 animals) than in asymptomatic animals (19 out of 31 animals). These results support the use of spleen instead of lymph node aspiration as the choice method for the parasitological diagnosis of the infection.
\end{abstract}

Key words: Leishmania chagasi - dog - diagnosis - Brazil

Zoonotic visceral leishmaniasis is caused by Leishmania chagasi/Leishmania infantum (Herwaldt 1999). The disease is endemic in American, European, and Asiatic countries (Pearson \& Sousa 1996, Herwaldt 1999) with new areas of infection dissemination being identified (Enserink 2000, Silva et al. 2001). The fact that dogs are considered the most important urban reservoir of $L$. chagasi makes canine visceral leishmaniasis (CVL) not only a major veterinary concern, but also a so far unsolved public health problem. Accurate diagnosis of canine infection by Leishmania is essential in veterinary practice, since the treatment of infected dogs includes 1520 daily injections of potentially toxic drugs, and in some countries, killing of infected animals is one of the measures applied in order to control the disease dissemination (Costa \& Vieira 2001, Moreno \& Alvar 2002). Dogs with symptomatic disease have fever, anemia, skin lesions, emaciation, hypergammaglobulinemia, hepato-splenomegaly, and lymphoadenomegaly, among other signs of the disease (Feitosa et al. 2000, Moreno \& Alvar 2002). The main pathological substrate of visceral leishmaniasis is amastigote multiplication in the mononuclear phagocytic

Financial support: Fundação Oswaldo Cruz (Papes II, grant 250.250.320) and Ministry of Science and Technology, Brazil (Brazilian National Research Council, CNPq, grant 52629/96-5 and Pronex)

${ }^{+}$Corresponding author. Fax: +55-71-356.3911. E-mail: sbarrouin@cpqgm.fiocruz.br

Received 30 September 2003

Accepted 6 February 2004 cells of internal organs such as spleen, liver, and bone marrow (Oliveira et al. 1993). In dogs, parasites are also found in peripheral lymph nodes and skin. The direct examination or cultivation of aspirates from spleen or bone marrow are the mainstay techniques used for the diagnosis of visceral leishmaniasis in both human beings and dogs. In fact, high sensitivity has been attributed to the parasitological diagnosis based on bone marrow or spleen examination in canine (Ashford et al. 1995) and human (Pearson \& Sousa 1996, Pintado et al. 2001) visceral leishmaniasis. Some authors, however, have used popliteal lymph node aspiration for parasite demonstration in CVL (Mathis \& Deplazes 1995, Ozensoy et al. 1998, SantosGomes et al. 2002). The reasons for using lymph node instead of spleen or bone marrow for diagnosis of CVL is the more peripheral location of lymph nodes (such as popliteo lymph node usually used in the procedure) and concerns about safety of the procedures (i.e. a possible risk of internal hemorrhage following spleen or liver puncture). However, no studies, to the best of our knowledge, have comparatively assessed the advantages and limitations of lymph node over internal organ examinations for the diagnosis of CVL.

In order to perform aspirative biopsies from lymph node or spleen, slight sedation must be induced in the animal, using pre-anesthetic drugs. Both procedures require technical training that do not involve different levels of difficulty. The main risks that can be anticipated for these procedures are spleen hemorrhage and popliteal neurovascular plexus lesion. In our group's practice, however, after more than 300 spleen puncture and more than 100 lymph node puncture, none of these intercurrences was observed, and only slight signs of discomfort were observed during lymph node puncture under sedation. 
In this study, the sensitivities of splenic and popliteal lymph node cultures for diagnosis of CVL were compared. We examined $64 \mathrm{dogs}$ from Monte Gordo, an endemic area for visceral leishmaniasis in the state of Bahia, Brazil. These animals varied in age (from 4 months to 9 years), sex (38 male, 26 female) and breed. All dogs had positive serological tests (ELISA or IF) for anti-Leishmania antibodies. Thirty one animals were asymptomatic, 26 had a few clinical signs of disease (oligosymptomatic), and 7 had multiple signs of visceral leishmaniasis (polysymptomatic), including emaciation, anemia, skin lesions around the nose, eyes, and on limbs, increase in size of lymph node and spleen and onicogriphosis (Table).

\section{TABLE}

Clinical categories and presence of parasite in culture lymph node and spleen aspirates of dogs with natural infection with Leishmania chagasi

\begin{tabular}{|c|c|c|c|c|c|}
\hline Clinical category & Spleen & $\begin{array}{l}\text { Lymph } \\
\text { node }\end{array}$ & $\begin{array}{l}\text { Spleen and } \\
\text { lymph } \\
\text { node }\end{array}$ & None & Total \\
\hline Asymptomatic & 16 & - & 3 & 12 & 31 \\
\hline Oligosymptomatic & 17 & - & 5 & 4 & 26 \\
\hline Polysymptomatic & 3 & 1 & 3 & - & 7 \\
\hline Total & 36 & 1 & 11 & 16 & 64 \\
\hline
\end{tabular}

The animals were tranquilized with an intravenous injection of $0.5 \mathrm{mg} / \mathrm{kg}$ of acepromazine and laid down on the right lateral side. Hairs were removed from the skin over the sites of puncture, and asepsis was done with a $2 \%$ alcohol-iodine solution. Splenic puncture was done in the left flank, near the edges of the last two ribs, using a $40 \mathrm{x}$ $12 \mathrm{~mm}$ needle and a $10 \mathrm{ml}$ syringe. The lymph node aspirates were done in the left popliteal lymph node using a 25 x $8 \mathrm{~mm}$ needle and a $10 \mathrm{ml}$ syringe. The spleen and lymph node samples (approximately 100-200 $\mu \mathrm{l}$ ) were cultured in a biphasic medium containing $1.5 \mathrm{ml}$ of blood-agar solid medium and $2 \mathrm{ml}$ of Schneider's medium supplemented with $20 \%$ of fetal calf serum. Cultures were maintained at $23^{\circ} \mathrm{C}$ and examined weekly under light microscopy during one month.

As shown in the Table, 16 out of the 64 dogs studied had negative spleen and lymph node cultures for Leishmania, $11(17.2 \%)$ had both spleen and lymph node positive cultures, $36(56.2 \%)$ had only spleen culture positive, and $1(1.6 \%)$ had only lymph node positive cultures. Therefore, sensitivity of spleen culture for diagnostic of $L$. chagasi was $73.4 \%$ (47/64), and of lymph node culture was $18.8 \%(12 / 64)$, both using serology as reference. Considering only the animals with infection confirmed in culture, the sensitivity of spleen culture for diagnostic of L. chagasi was $97.9 \%$, and of lymph node culture was $25 \%$. Positive spleen culture was more frequent $(\mathrm{p}=0.048$, Fisher's exact probability test) in symptomatic (28 out of 33 animals, $=84.8 \%)$ than in asymptomatic animals ( 19 out of 31 animals, $=61.3 \%$ ). There was no difference in the presence of positive lymph node culture between symptomatic and asymptomatic animals.
The results presented herein support the use of spleen instead of lymph node aspiration as the choice method for parasitological diagnosis of CVL caused by $L$. chagasi. Also, spleen puncture appeared to be better tolerated than the lymph node puncture, even in the most clinically affected dogs, exhibiting severe anemia and caquexia. The anatomic site where the popliteal lymph node is located is rich in nerves and nervous fibers, originated from the lombossacral plexus, which are susceptible to accidental traction and physical trauma during lymph node puncture. This fact may explain the transitory signs of pain demonstrated by most animals.

An interesting aspect of this work is the discrepancy of our findings on the sensitivity of lymph node aspirates with the experience reported by other authors in the literature (Mathis \& Deplazes 1995, Reale et al. 1999). Popliteal lymph node culture has been considered as a sensitive method for diagnosis (Mathis \& Deplazes 1995) and follow-up (Riera et al. 1999) of dogs with CVL. Reale et al. (1999) and Ozbel et al. (2000), on the other hand, found direct examination more effective than culture for the diagnosis of CVL, using total blood or lymph node aspirate. The low sensitivity of lymph node culture in the diagnosis of CVL observed is the study described herein cannot be attributed to poor parasite growth in culture due to culture conditions, since the sensitivity of spleen cultures performed under similar conditions and using specimens from the same animals was high. Contamination by microorganisms such as fungus or bacteria does not explain also the difference between spleen and lymph node culture sensitivities reported herein, since these cultures remained free of visible contamination for at least three weeks, a sufficient time for promastigote identification even under conditions of low inocula (Buffet et al. 1995). Another factor, which affects the ability to detect Leishmania in different tissues, is the host immunological status. Leishmania dissemination to unusual sites and high parasite burden are observed in hosts with severe impairment of the immunological response (Alvar et al. 1997). Generally, animals with clinical forms of CVL are in a more advanced stage of the infection and have depression of the cellular immune response to Leishmania (Rhalem et al. 1999). No differences in the frequency of positive lymph node culture between polysymptomatic animals and those with less severe disease, however, were observed in the present work. Finally, actual difference may exist in the tissue distribution of $L$. chagasi to the spleen and lymph node. Some studies reporting high sensitivity of lymph node examination for diagnosis of CVL were produced in Europe, where L. infantum is the infecting agent (Mathis \& Deplazes 1995, Reale et al. 1999). Evidences exist that $L$. chagasi and $L$. infantum are the same Leishmania species (Mauricio et al. 2002), probably introduced into the American Continent during European colonization. Hence, one of the possible explanations for the discrepancy between our data and that presented by those authors reporting high sensitivity of lymph node aspiration is the existence of variations in the Leishmania strains causing CVL in the Old and in the New World. In fact, the ability for disseminating and causing lesions at different sites such as skin and internal organs varies 
with the zymodeme expressed by different strains of $L$. infantum (Alvar et al. 1997, Sulahian et al. 1997). Similarly, and not yet well understood, differences in parasite distribution are also observed with strains of L. chagasi (Noyes et al. 1997). Further studies are necessary in order to identify the factors responsible for the putative differences in the ability of L. chagasi/L. infantum strains to disseminate to lymph nodes and also the molecular mechanisms involved in Leishmania tropism to different tissues.

\section{ACKNOWLEDGMENTS}

To Dr Moacir Paranhos Silva (Institute of Heath Sciences, UFBA), for his technical assistance on splenic punctures.

\section{REFERENCES}

Alvar J, Canavate C, Gutierrez-Solar B, Jimenez M, Laguna F, Lopez-Velez R, Molina R, Moreno J 1997. Leishmania and human immunodeficiency virus coinfection: the first 10 years. Clin Microbiol Rev 10: 298-319.

Ashford DA, Bozza M, Freire M, Miranda JC, Sherlock I, Eulalio C, Lopes U, Fernandes O, Degrave W, Barker Jr RH 1995. Comparison of the polymerase chain reaction and serology for the detection of canine visceral leishmaniasis. Am J Trop Med Hyg 53: 251-255.

Buffet PA, Sulahian A, Garin YJ, Nassar N, Derouin F 1995. Culture microtitration: a sensitive method for quantifying Leishmania infantum in tissues of infected mice. Antimicrob Agents Chemother 39: 2167-2168.

Costa CH, Vieira JB 2001. Changes in the control program of visceral leishmaniasis in Brazil. Rev Soc Bras Med Trop 34: 223-228.

Enserink M 2000. Infectious diseases. Has leishmaniasis become endemic in the U.S.? Science 290: 1881-1883.

Feitosa MM, Ikeda FA, Luvizotto MCR, Perri SHV 2000. Clinical aspects from dogs naturally infected with visceral leishmaniasis in Araçatuba, São Paulo State, Brazil. Clínica Veterinária 28: 36-44.

Herwaldt BL 1999. Leishmaniasis. Lancet 354: 1191-1199.

Mathis A, Deplazes P 1995. PCR and in vitro cultivation for detection of Leishmania spp. in diagnostic samples from humans and dogs. J Clin Microbiol 33: 1145-1149.

Mauricio IL, Stothard JR, Miles MA 2000. The strange case of Leishmania chagasi. Parasitol Today 16: 188-189.

Moreno J, Alvar J 2002. Canine leishmaniasis: epidemiological risk and the experimental model. Trends Parasitol 18: 399405.
Noyes H, Chance M, Ponce C, Ponce E, Maingon R 1997. Leishmania chagasi: genotypically similar parasites from Honduras cause both visceral and cutaneous leishmaniasis in humans. Exp Parasitol 85: 264-273.

Oliveira GGS, Santoro F, Sadigursky M 1993. The subclinical form of experimental visceral leishmaniasis in dogs. Mem Inst Oswaldo Cruz 88: 243-248.

Ozbel Y, Oskam L, Ozensoy S, Turgay N, Alkan MZ, Jaffe CL, Ozcel MA 2000. A survey on canine leishmaniasis in western Turkey by parasite, DNA and antibody detection assays. Acta Trop 74: 1-6.

Ozensoy S, Ozbel Y, Turgay N, Alkan MZ, Gul K, GilmanSachs A, Chang KP, Reed SG, Ozcel MA 1998. Serodiagnosis and epidemiology of visceral leishmaniasis in Turkey. Am J Trop Med Hyg 59: 363-369.

Pearson RD, Sousa AQ 1996. Clinical spectrum of leishmaniasis. Clinical Infectious Diseases 22: 1-13.

Pintado V, Lopez-Velez R 2001. Visceral leishmaniasis associated with human immunodeficiency virus infection. Enferm Infec Microbiol Clin 19: 353-357.

Reale S, Maxia L, Vitale F, Glorioso NS, Caracappa S, Vesco G 1999. Detection of Leishmania infantum in dogs by PCR with lymph node aspirates and blood. J Clin Microbiol 37: 2931-2935.

Rhalem A, Sahibi H, Guessouss-Idrissi N, Lasri S, Natami A, Riyad M, Berrag B 1999. Immune response against Leishmania antigens in dogs naturally and experimentally infected with Leishmania infantum. Vet Parasitol 81: 173184.

Riera C, Valladares JE, Gallego M, Aisa MJ, Castillejo S, Fisa R, Ribas N, Carrio J, Alberola J, Arboix M 1999. Serological and parasitological follow-up in dogs experimentally infected with Leishmania infantum and treated with meglumine antimoniate. Vet Parasitol 84: 33-47.

Santos-Gomes GM, Rosa R, Leandro C, Cortes S, Romão P, Silveira H 2002. Cytokine expression during the outcome of canine experimental infection by Leishmania infantum. Vet Immunol Immunopathol 88: 21-30.

Silva ES, Gontijo CM, Pacheco RS, Fiúza VO, Brazil RP 2001. Visceral leishmaniasis in the Metropolitan Region of Belo Horizonte, State of Minas Gerais, Brazil. Mem Inst Oswaldo Cruz 96: 285-291.

Sulahian A, Garin YJ, Pratlong F, Dedet JP, Derouin F 1997. Experimental pathogenicity of viscerotropic and dermotropic isolates of Leishmania infantum from immunocompromised and immunocompetent patients in a murine model. FEMS Immunol Med Microbiol 17: 131-138. 